This document is the Accepted Manuscript version of a Published Work that appeared in final form in ACS Applied Nano Materials, copyright (C) American Chemical Society after peer review and technical editing by the publisher.

To access the final edited and published work see: $\underline{\text { https://doi.org/10.1021/acsanm.8b00341 }}$ 


\title{
Spin Crossover in an Exfoliated 2-D Coordination Polymer and its Implementation in Thermochromic Films
}

\author{
Salvio Suárez-García, ${ }^{\dagger}$ Nayarassery N. Adarsh, ${ }^{\dagger}$ Gábor Molnár, ${ }^{\ddagger}$ Azzedine Bousseksou, ${ }^{\ddagger}$ Yann \\ Garcia, ${ }^{\S}$ Marinela M. Dîrtu, ${ }^{\S}$ Javier Saiz-Poseu, ${ }^{\dagger}$ Roberto Robles, ${ }^{\dagger}$ Pablo Ordejón ${ }^{\dagger}$ and Daniel \\ Ruiz-Molina*,† \\ ${ }^{\dagger}$ Institute of Nanoscience and Nanotechnology (ICN2), CSIC and BIST, Campus UAB, Bellaterra, o8193 Barcelona, \\ Spain. (D. R.-M. dani.ruiz@icn2.cat) \\ ${ }^{\ddagger}$ LCC, CNRS \& Université de Toulouse (UPS, INP), Toulouse, France. \\ ${ }^{\S}$ Univerité catholique de Louvain, Institute of Condensed Matter and Nanosciences Molecules, Solids and Reactivity \\ (IMCN/MOST), Place L. Pasteur 1, 1348 Louvain-la-Neuve
}

\begin{abstract}
Development of novel 2D materials with singular and thrilling properties has aroused large interest due to the novel unexpected applications that can be derived from there. In this sense, coordination polymers (CPs) have appeared as matching candidates thanks to their rational chemical design and the added-value properties given by the presence of metal ions. This is the case of switchable spin crossover systems that have been proposed as excellent candidates for data storage or sensing, among others. Here we report the delamination of crystals of the $2 \mathrm{D}$ spin crossover (SCO) $\left\{\left[\mathrm{Fe}(\mathbf{L 1})_{2}\right]\left(\mathrm{ClO}_{4}\right)_{2}\right\}_{\propto}(\mathbf{1}) \mathrm{CP}$ by liquid-phase exfoliation (LPE) in water. The application of this top-down technique results in the formation of flakes with controlled thicknesses, down to 1-2 nm thick (mostly mono- and bi-layer), that retain the chemical composition and SCO interconversion of the bulk material. Moreover, these flakes can be handled as stable colloidal dispersions for many days. This allows for a controlled transfer to solid substrates and the formation of thermochromic polymeric films as a proof-of-concept of device. These first results will definitely open new venues and opportunities for the investigation and future integration of these original switchable 2D materials in devices.
\end{abstract}

KEYWORDS: 2D coordination polymer, spin crossover, delamination, liquid-phase exfoliation, sonication, iron complex

\section{INTRODUCTION}

Coordination polymers (CPs) have been focus of research over the last years as an alternative approach to graphene and related $2 \mathrm{D}$ materials thanks to their rich synthetic chemistry, chemical flexibility and the presence of metal ions that add novel optical, magnetic and/or electrical properties. ${ }^{1-4}$ All these premises have already crystallized in different examples of $2 \mathrm{D}$ coordination polymers (CPs) relevant for the electrochemical detection of $\mathrm{H}_{2} \mathrm{O}_{2},{ }^{5}$ functional thin films, ${ }^{6}$ molecular sieve membranes, ${ }^{7}$ polymer composites for gas separation, ${ }^{8}$ catalysis 9,10 or photoluminescence. ${ }^{1,12}$ Despite these pioneering examples, control over the synthesis and applications of $2 \mathrm{D}$ materials based on coordination polymers still remains a significant research challenge. This is especially applicable for switchable systems, whose ability to interconvert between two different states under an external stimulus is envisaged as a promising alternative to classical inorganic materials in information processing and sensing. Spin crossover (SCO) metal complexes belong to this family of switchable molecular materials exhibiting a reversible interconversion between the so-called low spin
(LS) and high spin (HS) electronic configurations. ${ }^{13-17}$ The conversion between these two states can be triggered by various external stimuli such as temperature, pressure, light, X-ray irradiation, an intense magnetic field, electric field or the inclusion of guest molecules. ${ }^{18-20}$ The two spin states can be distinguished by their unique and different magnetic, optical, mechanical, spectroscopic and structural properties. Therefore, SCO materials have been proposed for numerous applications such as displays, ${ }^{21}$ memory devices, ${ }^{22}$ pressure and temperature sensors, ${ }^{23}$ gas sensors, ${ }^{24}$ nanothermometers ${ }^{25}$ optoelectronic devic$\mathrm{es}^{26}$ and actuators. ${ }^{27}$ So far, SCO materials have been studied as bulk powders or crystals ${ }^{28}$ and, more recently, as micro-/nanoscale thin films, nanoparticles ${ }^{29-31}$ or single molecules on the quest for their integration into functional devices. ${ }^{32-33}$ However, as far as we know, no example of a sheet-like SCO system has been described. The sole investigation in this direction concerns a self-assembled monolayer of SCO molecules, which has been recently studied by scanning tunneling microscopy (STM) revealing collective phenomena in $2 \mathrm{D}$ spin crossover layers. ${ }^{34}$ 


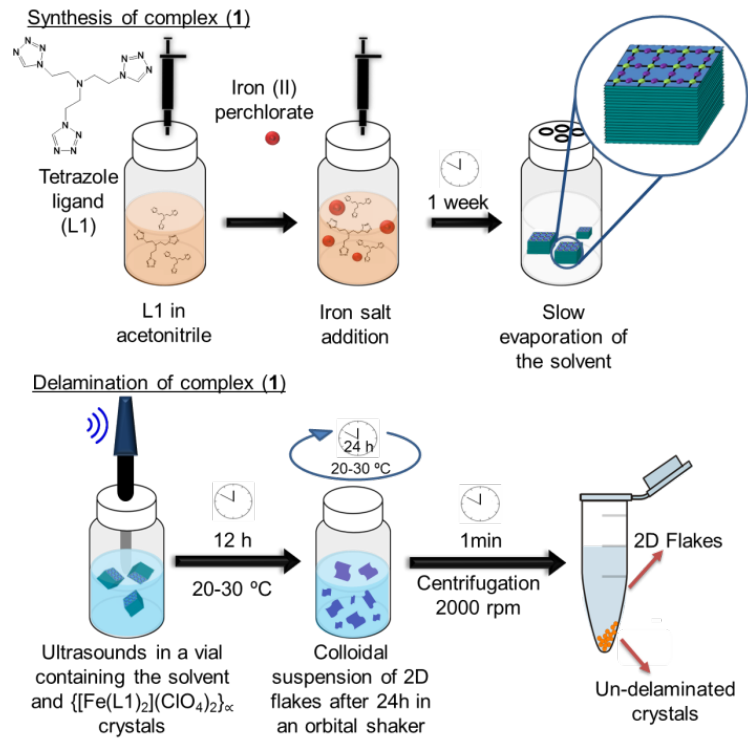

Figure 1. Scheme of the synthesis of the crystals (1) and their delamination by applying a top-down technique, which consists in a liquid-phase exfoliation (LPE) ultrasounds-assisted.

Herein we hypothesize that delamination of $2 \mathrm{D}$ bulk SCO crystals represents a successful approach for the unprecedented SCO flakes production method. As a representative example for these studies we have selected the $2 \mathrm{D}$ coordination polymer $\left\{\left[\mathrm{Fe}(\mathbf{L 1})_{2}\right]\left(\mathrm{ClO}_{4}\right)_{2}\right\}_{\propto}(\mathbf{1})$, which has already been shown to exhibit SCO. ${ }^{35-36}$ To achieve this objective, the two-step approach shown in Figure 1 was followed. It consisted in the growth of large crystals from solution and subsequent delamination by means of the well-known liquid-phase exfoliation method (LPE). ${ }^{37^{-}}$ 39 The process was optimized to obtain flakes thicknesses close to the single molecule level while retaining the SCO transition of the bulk crystals. Finally, the flakes were stabilized as colloidal dispersions that allow for their incorporation into free standing thermochromic thin films.

\section{EXPERIMENTAL SECTION}

CAUTION: perchlorate salts ${ }^{40}$ and tetrazole ligands ${ }^{41}$ are potentially explosive and should be handled with extreme care. The synthesis of tris(2-(1H-tetrazol-1-yl)ethyl)amine ligand (L1) and the coordination polymer $\left\{\left[\mathrm{Fe}(\mathrm{L1})_{2}\right]\left(\mathrm{ClO}_{4}\right)_{2}\right\}_{\propto} \quad(\text { complex }(\mathbf{1}))_{\text {were done following a }}$ previously reported procedure ${ }^{35}$ (for more detail, see Supporting Information, SI1)

Exfoliation process. Coordination polymer (1) was exfoliated in $2 \mathrm{D}$ flakes by using the LPE methodology. The process consisted in the addition of $1 \mathrm{mg}$ of the complex (1) into a vial containing $5 \mathrm{ml}$ of pure water. After 12 h, complex (1) showed a successful delamination of the layered crystal. For improving the exfoliation yield, the vial can be placed in an orbital shaker for $24 \mathrm{~h}$ (1-2D). Final step of centrifugation (200o rpm, $1 \mathrm{~min}$ ) was added in order to separate the unexfoliated crystals remaining in the pellet and the exfoliated flakes present in the superna- tant. For obtaining dried flakes powder, the solvent was evaporated slowly under vacuum conditions. FT-IR (deposited on silicon substrate by drop casting methodology): $3142\left(\mathrm{mb}\right.$, tetrazole $\left.v \mathrm{C}_{\mathrm{ttz}}-\mathrm{H}\right), 1500 \mathrm{~m}, 1438 \mathrm{~m}, 1361 \mathrm{~m}$, $1327 \mathrm{~m}, 126 \mathrm{om}, 1172 \mathrm{~m}, 1199 \mathrm{~s}, 1079$ (s, v perchlorate $\mathrm{Cl}-\mathrm{O})$ $988 \mathrm{~s}, 951 \mathrm{~s}, 87 \mathrm{om}, 785 \mathrm{~m}, 756 \mathrm{~m}, 711 \mathrm{~m}, 681 \mathrm{~s}, 662 \mathrm{~s}, 615$ (s, $\delta$ perchlorate $\mathrm{O}-\mathrm{Cl}-\mathrm{O}), 537 \mathrm{~m}, 505 \mathrm{~m} \mathrm{~cm}^{-1}$.

Transfer of (1-2D) to substrates. The main methodologies for transfer colloidal suspensions of flakes to substrate were drop casting and spin coating, using clean $\mathrm{Si}-$ wafers $(0.5 \times 0.5 \mathrm{~cm} 2)$ as a substrate. For the drop casting methodology, $15 \mu \mathrm{l}$ of colloidal suspension after exfoliation process was deposited on the silicon substrate. For the spin coating process, the substrate was rotated with a drop containing the flakes after the delamination process for $120 \mathrm{~s}$ at $3500 \mathrm{rpm}$. The substrates with (1-2D) flakes were dried under argon flow and stored in low humidity conditions under N2 atmosphere.

Preparation of (1-2D) films. Dried $2 \mathrm{D}$ flakes $(50 \mathrm{mg})$ were dispersed in a previously prepared poly(vinyl alcohol) (PVA) solution (6 mL, 20 wt.\%). The dispersion was sonicated during $2 \mathrm{~min}$ and slowly transferred to a plastic petri dish (diameter of $5 \mathrm{~cm}$ ). The (1-2D) SCO film was obtained after evaporation of the water at room temperature during $24 \mathrm{~h}$. The final film was easily removed from the petri dish once it was dry.

\section{RESULTS AND DISCUSSION}

Reaction of $\mathrm{Fe}\left(\mathrm{ClO}_{4}\right)_{2} \cdot \mathrm{xH}_{2} \mathrm{O}$ with a tris-tetrazole ligand (L1, Figure 2a) (1:2 metal:ligand ratio) in $\mathrm{CH}_{3} \mathrm{CN}$ and in contact with air, yielded yellowish crystals of complex (1) (for more details see Supporting Information, SI 1) whose SCO interconversion was fully confirmed (for more details see Supporting Information, SI 3). Even though complex (1) had already been reported, ${ }^{35}$ no crystallographic characterization was done at that time. For this reason, and due to the relevance for this work in such the structure and interaction between the $2 \mathrm{D}$ layers is crucial for a proper delamination process, single crystals of complex (1) suitable for X-ray diffraction were carefully grown, selected and analyzed.

Complex (1) crystallizes in a triclinic Pī space group with an asymmetric unit comprising one Fe(II) metal node (located on the center of inversion), one molecule of tetrazole ligand $\mathbf{L} \mathbf{1}$, and one perchlorate counter anion (for crystal data at different temperatures, see Supporting Information, Table $\mathrm{S}$ ). The resulting repetitive unit of $\mathrm{FeN}_{6}$ (Figure $2 \mathrm{~b}$, perchlorate remains uncoordinated) undergoes extended coordination leading to the formation of a $2 \mathrm{D}$-corrugated sheet. The layers are packed one on top of each other in an offset manner maintained by supramolecular interactions involving the tetrazole rings (see Figures $2 \mathrm{c}-2 \mathrm{~d}$ and Supporting Information SI 2 for more details). The Fe(II) metal node exhibits a slightly distorted octahedral geometry at $250 \mathrm{~K}[\mathrm{~N}-\mathrm{Fe}-\mathrm{N}=$ $\left.88.87(19)-91.13(19)^{\circ}\right]$ with the equatorial and apical positions of the $\mathrm{Fe}(\mathrm{II})$ coordinated by the tetrazole moieties of L1. 
a)

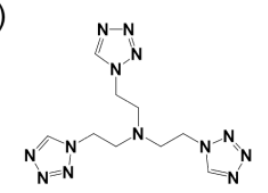

b)

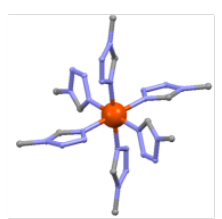

c)

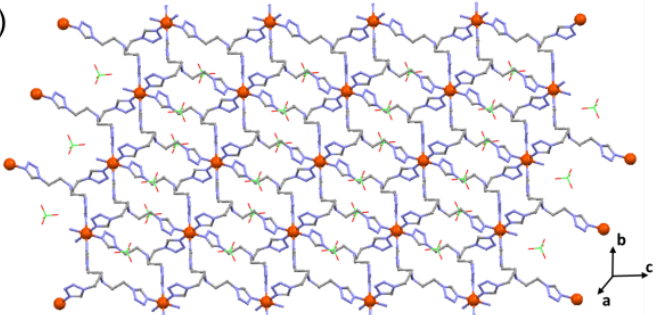

d)

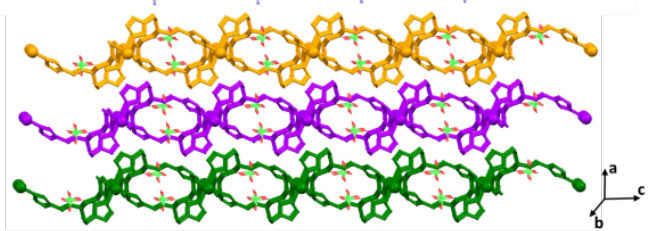

Figure 2. Crystallographic structure of complex (1). a) Tris-tetrazole ligand (L1); b) $\mathrm{FeN}_{6}$ chromophore (Fe(II) is represented in red); c) TOPOS view of the crystal structure of (1); each L1 ligand bridges three Fe(II) ions and, simultaneously, six $\mathbf{L} 1$ ligands are coordinated to one central atom; d) Packing of $2 \mathrm{D}$-corrugated sheets along the $b$ axis. The perchlorate anions occupy the interstitial spaces. This species is involved in hydrogen bonding and other weak interactions with the tetrazole rings of different layers (adjacent sheets are shown in orange, purple and green).

Before delamination, good-shaped crystals (average size $8.2 \pm 1.2 \mu \mathrm{m})$ were manually picked-up from the bulk sample (see Supporting Information, SI 4). X-ray powder diffraction (XRPD) data confirmed that selected crystals exhibit the same pattern compared with the simulated spectrum of a single crystal (see Supporting Information, Figure $\mathrm{S}_{5}$ ). The crystals were then dispersed on different solvents under sonication at room temperature for $12 \mathrm{~h}$ (for experimental details see Supporting Information, SI 1). Organic solvents such as dimethylformamide (DMF), acetone $\left(\left(\mathrm{CH}_{3}\right)_{2} \mathrm{CO}\right)$, chloroform $\left(\mathrm{CHCl}_{3}\right)$, toluene $(\mathrm{Tol})$ or tetrahydrofuran (THF) induce the chemical decomposition of the sample as FT-IR spectroscopy confirms (Supporting Information, Figure S6). In polar organic solvents such as methanol $(\mathrm{MeOH})$ and ethanol $(\mathrm{EtOH})$ the crystals are chemically stable though no flakes were found upon sonication. Overall, water turned out to be the most suitable solvent for the exfoliation process as the delaminated material is chemically stable at least for one week. This stability can be improved up to one month with a mixture of water/ethanol and up to several months under $\mathrm{N}_{2}$ atmosphere. The formation of colloidal suspensions containing exfoliated $2 \mathrm{D}$ flakes was optically confirmed by the observation of the Tyndall effect (Figure 3a). The laser beam can be observed through the suspensions; the scattered light indicates the presence of a col- loidal suspension of flakes, which is maintained after few weeks. Nevertheless, at this stage no control over the degree of delamination was achieved. With this aim, a time-dependent exfoliation experiment was ran in water and followed by combined dynamic light scattering (DLS) and atomic force microscopy (AFM) at three different sonication periods of 4,6 and $12 \mathrm{~h}$. The DLS measurements taken from samples at 4 and 6 h showed mean size diameters of $347 \pm 6 \mathrm{~nm}$ and $141 \pm 9 \mathrm{~nm}$, respectively (see Supporting Information, Figure $S_{7}$ ). Complementary AFM measurements of the same thin films upon deposition by spin coating on silicon surfaces revealed average thicknesses of $200 \mathrm{~nm}$ and $25 \mathrm{~nm}$, respectively (see Supporting Information, Figure S8). This would be in agreement with the obtaining of flakes a few hundreds and a few tens of layers, for 4 and $6 \mathrm{~h}$, respectively.
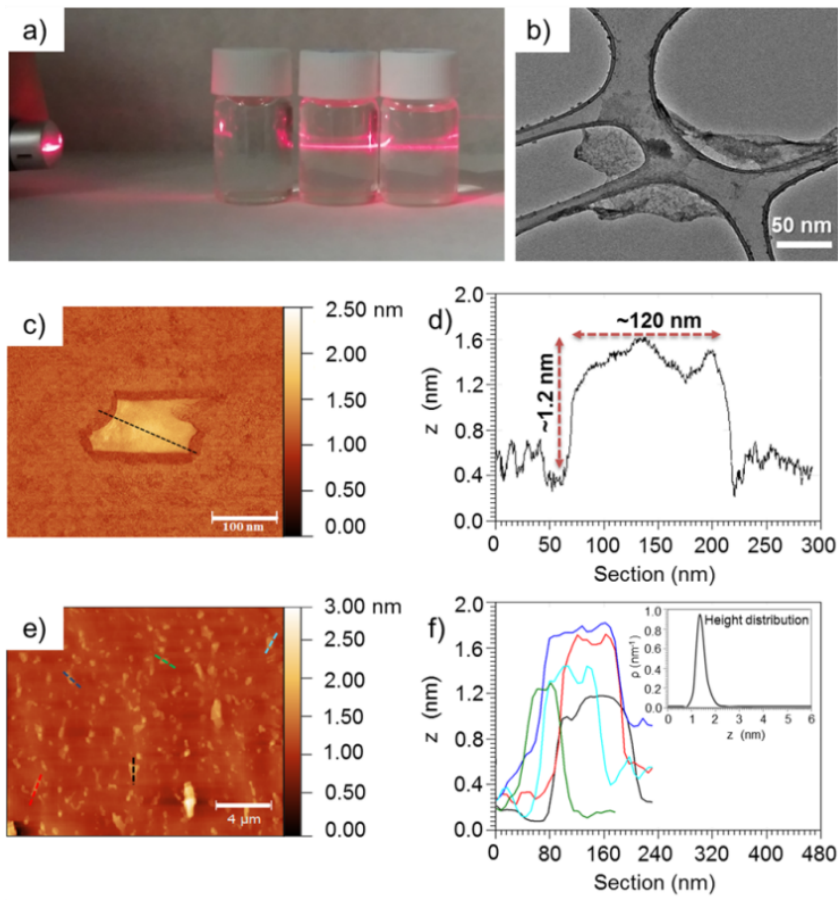

Figure 3. Morphological characterization of the flakes. a) Tyndall effect demonstration of the colloidal suspension in water. From left to right: the red laser beam remains invisible through the first vial, which contains the complex (1) before exfoliation. The scattered laser beam can be observed through the colloidal suspension of flakes immediately (in water, second vial) and few weeks (in water/ethanol mixture, third vial) after exfoliation; b) Transmission electron microscopy (TEM) image of a single flake, a few hundred nanometers width, obtained after transferring a drop of the same colloidal suspension to the grid; c) Tapping mode atomic force microscopy (AFM) topography (in ambient air) of a layer on silicon substrate; d) Height profile corresponding to the dashed line in panel c); e) General view of a tapping mode AFM topography (in ambient air) after the re-dispersion in water of the exfoliated flakes; f) Different height profiles corresponding to the dashed lines in panel e). Inset graph: Height profile distribution corresponding to the image showed in panel e). 
Sonication for a longer period of $12 \mathrm{~h}$ finally allowed for the formation of the desired flakes with average diameters of $86 \pm 7 \mathrm{~nm}$ (average Gaussian distribution centered on $100 \mathrm{~nm}$; see Supporting Information, Figures S7c-S8) and a narrow peak thickness distribution of $1-3 \mathrm{~nm}$, as confirmed by AFM. This would be in agreement with the presence of mostly mono- and bi-layer flakes (see Supporting Information, Figure S8). Representative transmission electron microscopy (TEM) and AFM images of one of the $2 \mathrm{D}$ flakes obtained after $12 \mathrm{~h}$ of sonication are shown in Figure $3 \mathrm{~b}-\mathrm{c}$. The chemical integrity of the flakes resulting after $12 \mathrm{~h}$ of sonication was also confirmed by different techniques
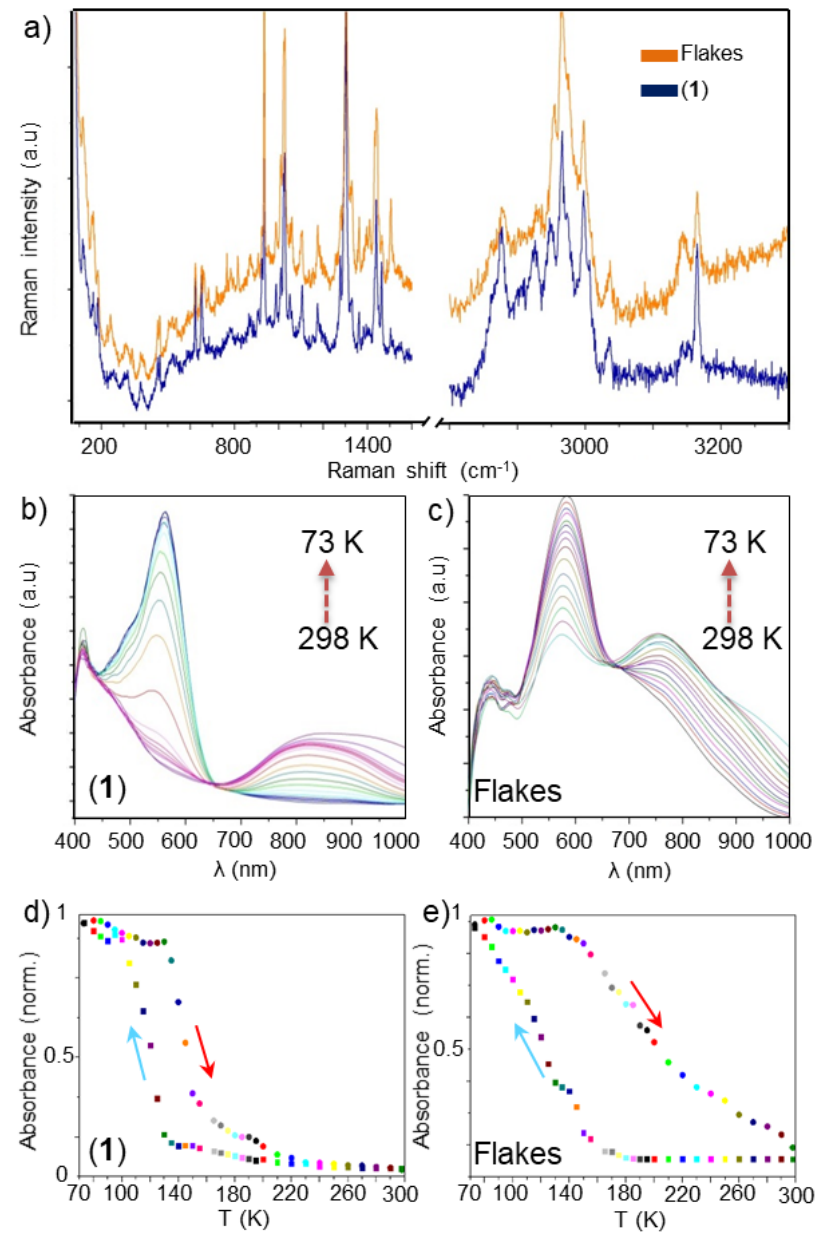

Figure 4. Bulk and flakes comparison. a) Room temperature Raman spectra (excited at $532 \mathrm{~nm}$ ) of the exfoliated flakes (orange) and bulk crystals (blue); b) Variabletemperature absorption spectra for the bulk crystals and c) flakes over the $73-298 \mathrm{~K}$ temperature range; d) Hysteresis loops obtained from temperature-depending absorbance spectra of complex (1) and e) for (1-2D) flakes on cooling ( $298 \mathrm{~K}$ to $73 \mathrm{~K}$ ) and heating ( $73 \mathrm{~K}$ to $298 \mathrm{~K}$ ) cycles. The spectra demonstrate the presence of hysteresis in both systems and the SCO. The temperatures recorded where from $73-80 \mathrm{~K}$ to $200 \mathrm{~K}$ (every $5 \mathrm{~K}$ ) and from $200 \mathrm{~K}$ to $298 \mathrm{~K}$ (every $10 \mathrm{~K}$ ). The blue arrow indicates the cooling process and the red arrow indicates the heating process.
X-ray photoelectron spectroscopy (XPS) measurements of both bulk and flakes are in excellent agreement (see Supporting Information, Figure S11-S14), confirming that the $\mathrm{Fe}(\mathrm{II})$ nodes retain their oxidation state along the exfoliation process. FT-IR (see Supporting Information, Figure $\mathrm{S}_{15}$ ) and Raman spectra (Figure 4a) also closely match for both bulk and flakes, except for some relative peak intensities. Additionally, the exfoliated flakes were deposited on silicon substrates and analyzed by XRD. The results show a remarkable decrease of the diffraction peak intensities (some of them even disappear) and their concomitant broadening, as expected upon reduction of the dimensionality. Their relative intensities do not reveal any remarkable orientation (for more details, see Supporting Information, Figure S16).

Once the chemical stability of complex (1) after the delamination process was demonstrated, the SCO behavior was studied for both bulk crystals and $2 \mathrm{D}$ flakes by means of UV-vis spectroscopy under different thermal cycles: cooling ( $298 \mathrm{~K}$ to $73 \mathrm{~K}$ ) and heating (73 K to $298 \mathrm{~K}$ ) (see Figure $4 \mathrm{~b}-\mathrm{c}$ ). At $73 \mathrm{~K}$, (with a complete LS state) the maximum absorbance of the crystals is at $\lambda=560 \mathrm{~nm}$. For the exfoliated flakes a red shift to lower energies is observed. A new maximum at $\lambda=585 \mathrm{~nm}$ is now observed, presumably due to enhanced light scattering. This absorption band is characteristic of the LS isomer which corresponds to ${ }^{1} \mathrm{~A}_{1} \quad{ }^{1} \mathrm{~T}_{1}$ ligand field transition of the LS Fe(II). This transition is accompanied by visible thermochromism, which goes from colorless (HS) to pink-violet (LS) in this case. An increase of the temperature induces a decrease of this LS absorption band until it almost disappears while a HS band appears and increases in intensity in the near infrared region. When the maximum absorbance of the LS peak was plotted for a whole temperature cycle (heatingcooling), hysteresis loops appeared for both bulk crystals and flakes (see Figure $4 \mathrm{~d}-\mathrm{e}$ ). For the bulk complex (1), the absorbance abruptly decreases around $150 \mathrm{~K}$ (during the heating process) and abruptly increases again upon cooling around $130 \mathrm{~K}$, with a hysteresis of $\sim 20 \mathrm{~K}$ (Figure $4 \mathrm{~d}$ ). Regarding 2D flakes, two main differences were observed for the heating-cooling cycles: I) the transition is not so abrupt but rather progressive over a broad range of temperatures, as expected considering the strong phononic dependence of SCO systems and II) a broader hysteresis associated to the smoother interconversion process is observed (Figure 4e). Finally, for both samples consecutive fully reversible cooling-heating cycles were done thus confirming the stability and reproducibility of the SCO interconversion (for more details, see Supporting Information, SI 8 and Figure S18-S19). Additionally to the optical measurements, the spin-transition of (1-2D) flakes was also studied by other means: temperature-variable magnetization and Raman spectroscopy. Nevertheless, due to the lack of resolution of these techniques, it was necessary to increase the amount of material for obtaining enough signal (for a more detailed discussion, see Supporting Information, SI 9).

Once the exfoliated and dried flakes were measured and properly characterized, it was possible to redisperse them 
again in pure $\mathrm{H}_{2} \mathrm{O}$. The formation of de novo colloidal suspensions with average diameters of $90 \pm 4 \mathrm{~nm}$ was observed (see Supporting Information, Figure S9). The redispersed flakes were then deposited on Si substrates and analyzed by AFM (see Supporting Information, Figure Sio) revealing the same uniform narrow height distribution peaks around $1-2 \mathrm{~nm}$ (i.e. the same thickness) as observed for the exfoliated flakes just after sonication (Figure 3e). These results confirmed the stability of the flakes along the manipulation and physical measurements.

Additional ab initio density functional theory (DFT) calculations were done to get a first approximation of the SCO behavior on the translation from the bulk crystals to the flakes. For this, the experimental bulk structure (Figure $5 \mathrm{a}$ ) at two temperatures ( $110 \mathrm{~K}$ and $250 \mathrm{~K}$ ) was considered as a reference. For the low temperature structure (110 $\mathrm{K})$, the LS configuration $(\mathrm{S}=\mathrm{O})$ is more stable than the HS one $(\mathrm{S}=2)$ by $2.027 \mathrm{eV}$, whereas for the higher temperature structure $(250 \mathrm{~K})$ the HS state is $0.281 \mathrm{eV}$ lower in energy than the LS one. The Projected Densities of States (PDOS) (Figure $5 \mathrm{~b}$ ) for both configurations show the expected $\mathrm{Fe}$ electronic structure, confirming that the Fe metal ions are isolated, exhibiting only interactions with adjacent $\mathrm{N}$ atoms. The additional calculations performed in a single monolayer, fixing the coordinates to the bulk positions (Figure $5 \mathrm{C}$ ), revealed the similarities between bulk crystals and $2 \mathrm{D}$ flakes. In the case of a single flake, the low temperature structure (110 K) shows a LS state $2.047 \mathrm{eV}$ lower in energy than the HS configuration. For the high temperature structure $(250 \mathrm{~K})$ the HS state is more stable by $0.273 \mathrm{eV}$. The Fe PDOS in the monolayer (Figure $5 \mathrm{~d}$ ) is quite similar to the bulk case, showing that the intralayer interaction is hardly affecting the Fe d states. Both for bulk and monolayer, the electronic structure calculations confirm the experimentally identified spin state for low $(110 \mathrm{~K})$ and high $(250 \mathrm{~K})$ temperature structures and the existence of a spin transition when decreasing the temperature of the complex.

Finally, thermochromic free standing films were obtained by drop casting a aqueous colloidal dispersion of the flakes containing poly(vinyl alcohol) (PVA) (for more experimental details see Supporting Information, SI 1). As shown in Figure 6, at room temperature the films are translucent (slightly yellowish), characteristic of the high spin isomer. Upon cooling down to liquid nitrogen temperature $(77 \mathrm{~K})$, the color of the films changed to an intense pink/purple tonality, characteristic of the low spin isomer. The reproducibility and reversibility of the SCO in films were confirmed by repeated complete cooling and heating cycles. Additionally, several thin films were prepared in order to confirm the viability of the film production method containing $2 \mathrm{D}$ flakes. As a representative example, a time-dependent se-quence of photograms obtained after removal of a film from liquid nitrogen and exposition to room tempera-ture is shown in Figure 6b; the interconversion is fast, taking place in a few seconds (see video in Supporting Information)..

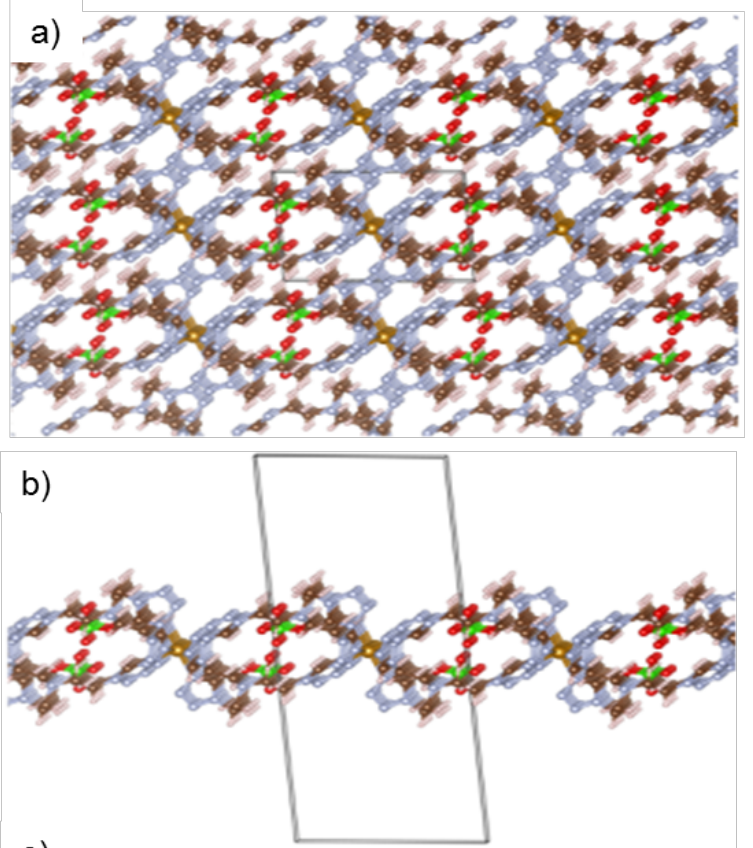

c)
$110 \mathrm{~K}$

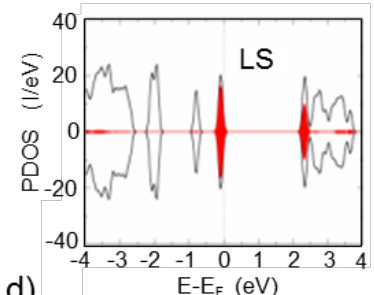

d)

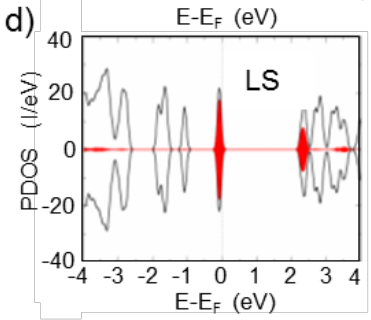

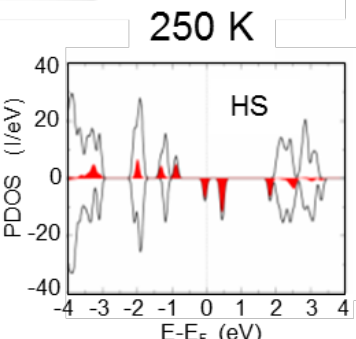

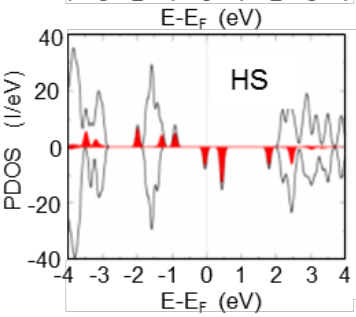

Figure 5. Theoretical model. a) Experimental bulk structure where the unit cell is shown; b) Structure for the monolayer where the atom positions are fixed to the bulk configuration. The unit cell is shown. PDOS for the c) bulk and d) monolayer using the experimental structure obtained at two different temperatures: $110 \mathrm{~K}$ (left) and $250 \mathrm{~K}$ (right). The red shaded area shows the Fe states.

As a representative example, a time-dependent sequence of photograms obtained after removal of a film from liquid nitrogen and exposition to room temperature is shown in Figure 6b; the interconversion is fast, taking place in a few seconds (see video in Supporting Information).Worth to mention, the chromogenic changes observed are homogenous in the whole film, most likely being an indication of the good dispersion of the flakes within the polymer. Just to confirm this assumption, different sections of the film were analyzed by SEM after breaking the material under cryogenic conditions. The result is shown in Figure 6c. As expected, a good distribution of the films, without relevant phase segregation processes was found. 

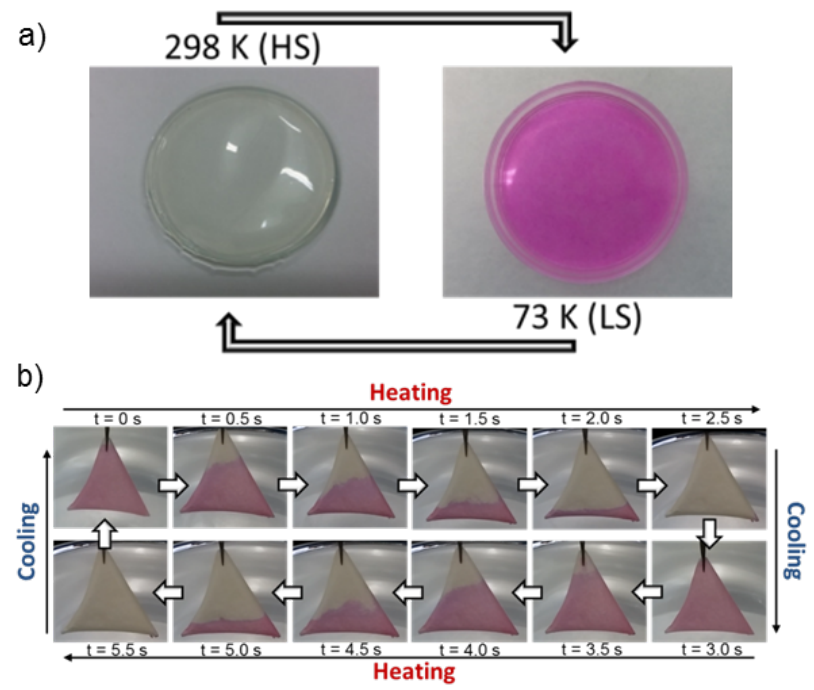

c)

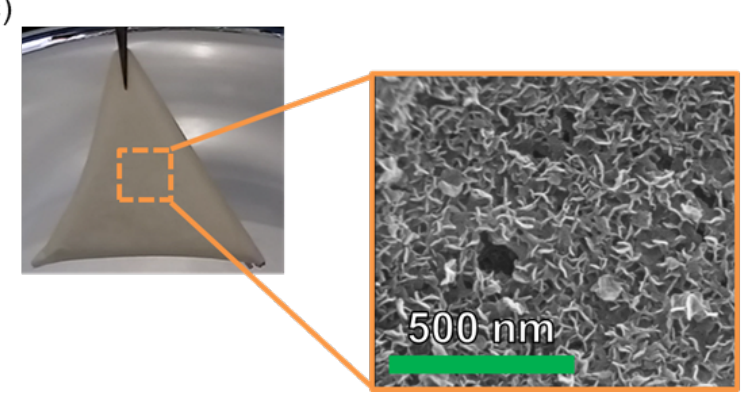

Figure 6. $2 \mathrm{D}$ flakes in a polymeric matrix. a) Films of $2 \mathrm{D}$ flakes on a poly(vinyl alcohol) (PVA) at room temperature (HS, left) and $77 \mathrm{~K}$ (LS, right); b) Complete cycles of cooling and heating of a PVA film containing the $2 \mathrm{D}$ exfoliated flakes; c) SEM image of a film containing the $2 \mathrm{D}$ flakes on PVA matrix.

\section{CONCLUSIONS}

In summary, $2 \mathrm{D}$ sheet-like spin crossover flakes with controlled thicknesses have been successfully obtained following the LPE approach. It has been shown by both experimental and theoretical means that the flakes retain the chemical properties of the bulk material and, what is even more relevant, the SCO behavior. However, as the spin transition is known to be remarkably sensitive to slight changes of the intermolecular interactions, the SCO transition of the flakes takes place more gradually over a broader temperature range.

Interestingly, the flakes can be handled as stable colloidal suspensions for many days, what allows for their transfer to different substrates of technological relevance. In this way, the flakes have been homogenously dispersed into PVA, resulting in the formation of free standing thin films with thermochromic behavior. Although the synthetic methodology for $2 \mathrm{D}$ coordination polymers is in its early stage, these results propel $2 \mathrm{D}$ SCO flakes as robust nanoplatforms to translate highly environmentally sensitive SCO systems into future SCO hybrid devices, in a reproducible and controlled manner.

\section{ASSOCIATED CONTENT}

Supporting Information. Experimental materials and methods, crystallographic information, detailed information of the SCO characterization for bulk crystals and additional physical and morphological characterization of $2 \mathrm{D}$ flakes can be found in the Supporting Information. Additionally, a video with the behavior of the thin films is provided. [CCDC No. 1534951-1534953 contains the supplementary crystallographic data for this paper. These data can be obtained free of charge from The Cambridge Crystallographic Data Centre via www.ccdc.cam.ac.uk/data_request/cif.]

\section{AUTHOR INFORMATION}

\section{Corresponding Author}

* Daniel Ruiz-Molina, e-mail: dani.ruiz@icn2.cat

\section{Author Contributions}

The manuscript was written through contributions of all authors. All authors have given approval to the final version of the manuscript.

\section{Notes}

The authors declare no competing financial interest.

\section{ACKNOWLEDGMENT}

This work was supported by projects MAT2015-70615-R, CTQ2015-65439-R and FIS2015-64886-C5-3-P from the Spanish Government funds and by European Regional Development Fund (ERDF). Funded by the CERCA Program/Generalitat de Catalunya. ICN2 is supported by the Severo Ochoa program from Spanish Ministry of Economy, Industry and Competitiveness (MINECO, Grant SEV-20130295). Funded by the project 2014-SGR-301 (Generalitat de Catalunya). This work was supported by the Fonds National de la Recherche Scientifique-FNRS (PDR T.0102.15) and COST actions CM1305 and CA15128. M. M. D is a chargé de recherches from the F.R.S.-FNRS. S.S.-G. acknowledges the support from MINECO BES-2015-071492 grant. R.R. and P.O. acknowledge support from EU H2020-EINFRA-5-2015 MaX Center of Excellence (Grant 676598).

\section{REFERENCES}

(1) Mas-Balleste, R.; Gomez-Navarro, C.; Gomez-Herrero, J.; Zamora, F. 2D Materials: to Graphene and Beyond. Nanoscale 2011, 3, 20-30.

(2) Colson, W. R.; Dichtel, J. W. Rationally Synthesized TwoDimensional Polymers. Nat. Chem. 2013, 5, 453-465.

(3) Rodríguez-San-Miguel, D.; Amo-Ochoa, P.; Zamora, F. MasterChem: cooking 2D-polymers. Chem. Commun. 2016, 52, 41134127.

(4) Rodenas, T.; Luz, I.; Prieto, G.; Seoane, B.; Miro, H.; Corma, A.; Kapteijn, F.; Llabrés-Xamena, F. X.; Gascon, J. Metal-Organic Framework Nanosheets in Polymer Composite Materials for Gas Separation. Nat. Mater. 2015, 14, 48-55.

(5) Wang, Y.; Zhao, M.; Ping, J.; Chen, B.; Cao, X.; Huang, Y.; Tan, Ch.; Ma, Q.; Wu, Sh.; Yu, Y.; Q. Lu, Chen, J.; Zhao, W.; Ying, Y.; Zhang, H. Bioinspired Design of Ultrathin 2D Bimetallic Metal-Organic-Framework Nanosheets Used as Biomimetic Enzymes. Adv. Funct. Mater. 2016, 28, 4149-4155.

(6) Xu, G.; Yamada, T.; Otsubo, K.; Sakaida, S.; Kitagawa, H. Facile "Modular Assembly" for Fast Construction of a Highly 
Oriented Crystalline MOF Nanofilm. J. Am. Chem. Soc. 2012, 134, 16524-16527.

(7) Peng, Y.; Li, Y.; Ban, Y.; Jin, H.; Jiao, W.; Liu, X.; Yang, W. Metal-Organic Framework Nanosheets as Building Blocks for Molecular Sieving Membranes. Science 2014, 346, 1356-1359.

(8) Zhang, Y.; Feng, X.; Yuan, S.; Zhou, J.; Wang, B. Challenges and Recent Advances in MOF-Polymer Composite Membranes for Gas Separation. Inorg. Chem. Front. 2016, 3, 896-909.

(9) Zhan, G.; Zeng, H. C. Synthesis and Functionalization of Oriented Metal-Organic-Framework Nanosheets: Toward a Series of 2D Catalysts. Adv. Funct. Mater. 2016, 26, 3268-3281.

(10) Dong, R.; Pfeffermann, M.; Liang, H.; Zheng, Z.; Zhu, X.; Zhang J.; Feng, X. Large-Area, Free-Standing, Two-Dimensional Supramolecular Polymer Single-Layer Sheets for Highly Efficient Electrocatalytic Hydrogen Evolution. Angew. Chem. Int. Ed. Engl. 2015, 54, 12058-12063.

(11) Hermosa, C.; Horrocks, B. R.; Martínez, J. I.; Liscio, F.; Gómez-Herrero, J.; Zamora, F. Mechanical and Optical Properties of Ultralarge Flakes of a Metal-Organic Framework with Molecular Thickness. Chem. Sci. 2015, 6, 2553-2558.

(12) Araki, T.; Kondo, A.; Maeda, K. The First Lanthanide Organophosphonate Nanosheet by Exfoliation of Layered Compounds. Chem. Commun. 2013, 49, 552-554.

(13) Gütlich, P.; Goodwin, H. A. Spin Crossover in Transition Metal Compounds I-III. In Topics in Current Chemistry; Meijere, A. de; Houk, K.N.; Kessler, H.; Lehn, J.-M.; Ley, S.V.; Schreiber, S.L.; Thiem, J.; Trost, B.M.; Vögtle, F.; Yamamoto, H., Eds.; Springer-Verlag, Berlin, Germany, 2004; pp. 59-9o.

(14) Bousseksou, A.; Molnár, G.; Salmon, L.; Nicolazzi, W. Molecular Spin Crossover Phenomenon: Recent Achievements and Prospects. Chem. Soc. Rev. 2011, 40, 3313-3335.

(15) Gütlich, P.; Hauser, A.; Spiering, H. Thermal and Optical Switching of Iron(II) Complexes. Angew. Chem. Int. Ed. Engl. 1994, 33, 2024-2054.

(16) Garcia, Y.; Adarsh, N. N.; Naik, A. D. Crystal Engineering of FeII Spin Crossover Coordination Polymers Derived from Triazole or Tetrazole Ligands. Chimia 2013, 67, 411-418.

(17) Halcrow, M. In Spin-crossover Materials: Properties and Applications; Halcrow, M., Eds.; Wiley, Germany, 2013; pp. 147169.

(18) Kumar, S.K., Ruben, M. Emerging Trends in Spin Crossover (SCO) Based Functional Materials and Devices. Coord. Chem. Rev. 2017, 346, 176-205.

(19) Wang, J.-L., Liu, Q. Meng, Y.-S., Zheng, H., Zhu, H.-L., Shi, Q., Liu, T. Synergic on/off Photoswitching Spin State and Magnetic Coupling between Spin Crossover Centers. Inorg. Chem., 2017, 56, 10674-1068o.

(20) Wang, H.-Y., Ge, J.-Y., Hua, C., Jiao, C.-Q, Wu, Y., Leong, C. F., D’Alessandro, D. M., Liu, T. Zuo, J.-L. Photo- and Electronically Switchable Spin-Crossover Iron(II) Metal-Organic Frameworks Based on a Tetrathiafulvalene Ligand. Angew. Chem. Int. Ed. Engl. 2017, 56, 5465-5470.

(21) Létard, J. F.; Guionneau, P.; Goux-Capes, L. Spin Crossover in Transition Metal Compounds III. In Towards Spin Crossover Applications; Meijere, A. de; Houk, K.N.; Kessler, H.; Lehn, J.-M.; Ley, S.V.; Schreiber, S.L.; Thiem, J.; Trost, B.M.; Vögtle, F.; Yamamoto, H., Eds.; Springer-Verlag, Berlin, Germany, 2004; pp. 221-249.

(22)Kahn, O.; Martinez, C. J. Spin-Transition Polymers: From Molecular Materials Toward Memory Devices. Science 1998, 279, 44-48.
(23) Linares, J.; Codjovi, E.; Garcia, Y. Pressure and Temperature Spin Crossover Sensors with Optical Detection. Sensors 2012, 12, 4479-4492.

(24) Ohba, M.; Yoneda, K.; Agusti, G.; Muñoz, M. C.; Gaspar, A. B.; Real, J. A.; Yamasaki, M.; Ando, H.; Nakao, Y.; Sakaki, S.; Kitagawa, S. Bidirectional Chemo-Switching of Spin State in a Microporous Framework. Angew. Chem. Int. Ed. Engl. 2009, 48, 4767-4771.

(25) Kraieva, O.; Quintero, C.M.; Suleimanov, I.; Hernandez, E.M.; Lagrange, D.; Salmon, L.; Nicolazzi, W.; Molnár, G.; Bergaud, C.; Bousseksou, A. High Spatial Resolution Imaging of Transient Thermal Events Using Materials with Thermal Memory. Small, 2016, 12, 6325-6331.

(26) Matsuda, M.; Kiyoshima, K.; Uchida, R.; Kinoshita, N.; Tajima, H. Characteristics of Organic Light-Emitting Devices Consisting of Dye-Doped Spin Crossover Complex Films. Thin Solid Films 2013, 531, 451-453.

(27) Shepherd, H. J.; Guralskiy, I. A.; Quintero, C. M.; Tricard, S.; Salmon, L.; Molnár, G.; Bousseksou, A. Molecular Actuators Driven by Cooperative Spin-State Switching. Nat. Commun. 2013, 4, 2607-2616.

(28) Jian-Ping, M.; Shao-Chen, L.; Chao-Wei, Z.; Xiao-Meng, Z.; Chuan-Zhi, S.; Yu-Bin, D. Reversible visual thermochromic coordination polymers via single-crystal-to-single-crystal transformation. CrystEngComm, 2014, 16, 304-307.

(29) Dugay, J., Giménez-Marqués, M., Kozlova, T., Zandbergen, H. W., Coronado, E. and van der Zant, H. S. J. Spin Switching in Electronic Devices Based on 2D Assemblies of Spin-Crossover Nanoparticles. Adv. Mater., 2015, 27, 1288-1293.

(30) Giménez-Marqués, García-Sanz de Larrea, M.L., Coronado, E. Unravelling the Chemical Design of Spin-Crossover Nanoparticles Based on Iron(II)-Triazole Coordination Polymers: Towards a Control of the Spin Transition. J. Mater. Chem. C, 2015, 3, 7946-7953.

(31) Volatron, F., Catala, L. Rivière, E., Gloter, A., Stéphan, O., Mallah, T. Spin-Crossover Coordination Nanoparticles. Inorg. Chem., 2oo8, 47, 6584-6586.

(32) G. Gopakumar, T.; Matino, F.; Naggert, H.; Bannwarth, A.; Tuczek, F.; Berndt, R. Electron-Induced Spin Crossover of Single Molecules in a Bilayer on Gold. Angew. Chem. Int. Ed. Engl. 2012, 51, 6262-6265.

(33) Miyamachi, T.; Gruber, M.; Davesne, V.; Bowen, M.; Boukari, S.; Joly, L.; Scheurer, F.; Rogez, G.; Kazu, Y. T.; Ohresser, P.; Beaurepaire, E.; Wulfheke, W. Robust spin crossover and memristance across a single molecule. Nat. Commun. 2012, 3, 938-943.

(34) Bairagi, K.; Iasco, O.; Bellec, A.; Kartsev, A.; Li, D.; Lagoute, J.; Chacon, C.; Girard, Y.; Rousset, S.; Miserque, F.; Dappe, Y. J.; Smogunov, A.; Barreteau, C.; Boillot, M. -L.; Mallah, T.; Repain, V. Molecular-Scale Dynamics of Light-Induced Spin Cross-Over in a Two-Dimensional Layer. Nat. Commun. 2016, 7, 12212-12219.

(35) Adarsh, N. N., Dîrtu, M. M., Rotaru, A., Garcia, Y. ${ }^{57} \mathrm{Fe}$ Mössbauer spectroscopy study of a $2 \mathrm{D}$ spin transition coordination polymer built from a tris-1R-tetrazole ligand, Hyperfine Interactions, 2017, 238:60.

(36) Bronisz, R. PhD thesis 1999, University of Wroclaw.

(37) Amo-Ochoa, P.; Welte, L.; Gonzalez-Prieto, R.; Sanz Miguel, P. J.; Gomez-Garcia, C. J.; Mateo-Marti, E.; Delgado, S.; Gomez-Herrero, J.; Zamora, F. Single Layers of a Multifunctional Laminar $\mathrm{Cu}(\mathrm{I}, \mathrm{II})$ Coordination Polymer. Chem. Commun. 2010, $46,3262-3264$. 
(38) Sakamoto, R.; Takada, K.; Pal, T.; Maeda, H.; Kambe, T.; Nishihara, H. Coordination Nanosheets (CONASHs): Strategies, Structures and Functions. Chem. Commun. 2017, 53, 5781-5801.

(39) Li, P.-Z.; Maeda, Y.; Xu, Q. Top-down Fabrication of Crystalline Metal-Organic Framework Nanosheets. Chem. Commun. 2011, $47,8436-8438$.

(40) Wolsey, W. C. J. Safety in the Chemical Laboratory. Chem. Educ. 1973, 50, A335-A337.

(41) Benson, F. R. The Chemistry of the Tetrazoles. Chem. Rev. 1947, 41, 1-61. 
SYNOPSIS TOC Exfoliation under sonication of bulk crystals of complex $\left\{\left[\mathrm{Fe}(\mathbf{L 1})_{2}\right]\left(\mathrm{ClO}_{4}\right)_{2}\right\} \propto(\mathbf{1})$ results in the formation of flakes that not only retain spin crossover (SCO) behavior of the bulk crystals, but also can be handled and stored as stable colloidal dispersions while being easily transferred to different substrates, such as a thermochromic free standing thin film, opening the way to the development of novel $2 \mathrm{D}$ switchable molecular devices.

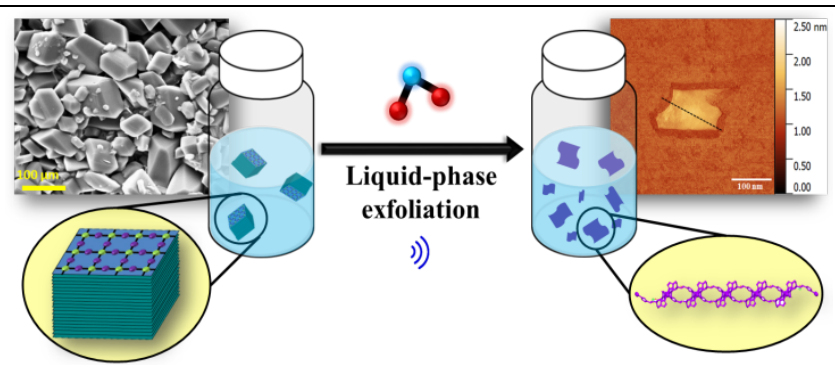

